\title{
Nanodentistry: At The Cross Road Of A New Era
}

Dr. Mayur Anand M.D.S Reader*,

Dr. Bina Anand M.D.S Reader *,

Dr. Kirti Anand M.D.S

Sr. Lecturer *,
Dr. Vinay Kumar G. M.D.S Professor,

Dept. of Prosthodontics College of Dental Sciences, Davangere.

* Dept. of Prosthodontics

Uttaranchal Dental and Medical Research Institute, Dehradun.

\section{INTRODUCTION}

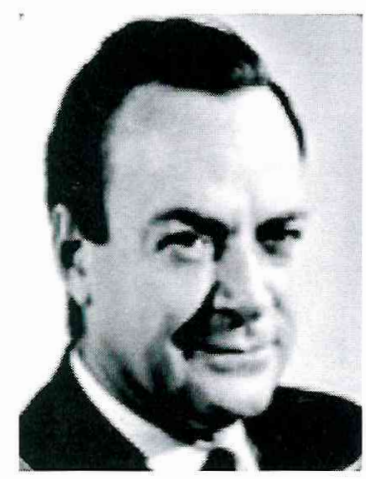

Figure. 1 : Richard P Faynman
Science is the fuel for the engine of technology. Today the revolutionary development of nanotechnology has become the most highly energized discipline in science and technology. The vision of nanotechnology was introduced in 1959 by late Nobel Physicist Richard P Faynman (Fig. 1) in a dinner talk where he said, "there is plenty of room at the bottom" and proposed employing machine tools to make smaller machine tools, these are to be used in turn to make still smaller machine tools, and so on all the way down to the atomic level. " Nanotechnology" term was coined by Prof. Kerie Drexler. Nano is derived from the greek word "vavo $\zeta$ " which means dwarf. A nanometer is 10-9 meter, or one-billionth of a meter. It is engineering at the molecular scale. ${ }^{2}$ Nanotechnology is defined as the research and development of materials, devices, and systems exhibiting physical, chemical, and biological properties that are different from those found on a large scale (matter smaller than scale of things like molecules and viruses). The basic concept is to create such small machines/ro-

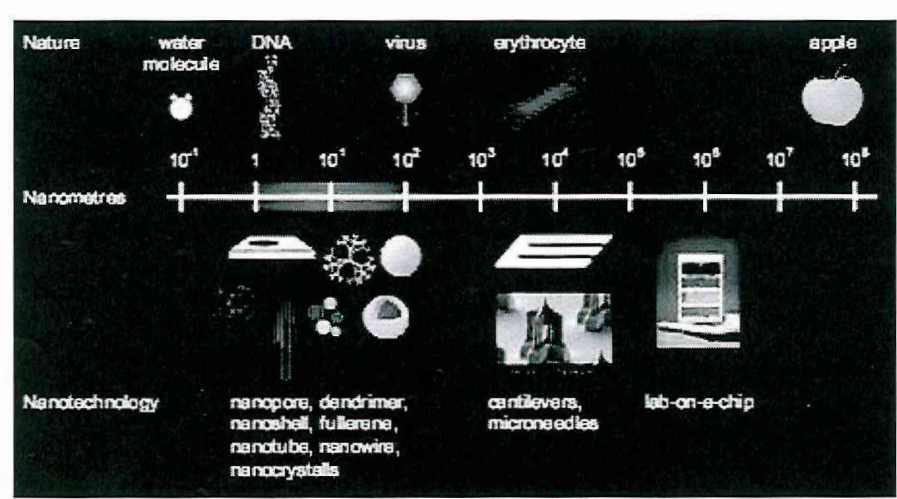

Figure 2 : Depicting size of Nanoparticles bots and products which will work at the atomic level. ${ }^{3}$ (Fig. 2)

\section{APPROACHES TO NANOTECHNOLOGY}

Bottom up approach: These seek to arrange smaller components into more complex assemblies.

Top down approach: These seek to create smaller devices by using larger ones to direct their assembly. Medical nanorobots have been proposed for gerontological applications, in pharmaceutical research and clinical diagnosis. Other applications include mechanically reversing atherosclerosis, improving respiratory capacity, enabling near instantaneous hemostasis, supplementing the immune system rewriting or replacing DNA sequences in cells, repairing brain damage and resolving gross cellular insults, whether caused by "irreversible" processes or by cryogenic storage of biological tissues.

A unique aspect of nanotechnology is the vastly increased ratio of surface area to volume present in many nanoscale materials which opens new possibilities in surface-based science, such as catalysis. Materials reduced to the nanoscale can suddenly show very different properties compared to what they exhibit on a macroscale, enabling unique applications. For instance, opaque substances become transparent (copper); inert materials become catalysts (platinum); stable materials turn combustible (aluminum); solids turn into liquids at room temperature (gold); insulators become conductors (silicon).

Development of 'Nanodentistry' will make possible the maintenance of near perfect oral health through the use of nanomaterials, biotechnology including tissue engineering and nanorobotics. The nanorobotics functions may be controlled by an onboard nanocomputer that executes preprogrammed instructions in response to local sensor stimuli. ${ }^{1}$ Role of nanotechnology in dentistry includes: Diagnostic science, dental material sciences, preven- 
tive dentistry, dental surgical procedures.

\section{NANOSTRUCTURES FOR DENTAL APPLI- CATION: ${ }^{4}$}

Nanoparticles: Molecular units typically defined as having diameters of between 0.1 and $100 \mathrm{~nm}$. Currently used in resin based composites.

Nanorods: Chen and colleagues have synthesized enamel-prism-like hydroxyapatite (HA) nanorods that have exhibited self assembly properties.

Nanospheres: Nanosphere assembly in conjunction with calcium phosphate deposition and amelogenin nanochain assembly lead to the formation and directional orientation of HA crystals that compose enamel's hard-tissue mineral phase.

Nanotubes: Titanium oxide nanotubes have shown to accelerate the kinetics of hydroxyapatite formation, mainly in a context of bone-growth applications for dental implant coatings. More recently, modified single-walled carbon nanotubes (SWCNTs) have been shown to improve flexural strength of composites.

Nanofibers: More recently, nanofibers have been used to generate ceramics containing hydroxyapatite and fluor-hydroxyapatite.

Dendrimers and dendritic copolymer: Combinations of specific polymers to optimize efficacy of restorative applications have been reported.

Presently available products in dentistry are:

Nanocomposites - Nanocomposites (Fig. 3) were developed to be used in all areas of the mouth with high initial polish and superior polish retention (typical of microfills) as well as excellent mechanical properties suitable for high stress bearing restorations (typical of hybrid). Two types of nanofillers are: nanomeric(NM)- these are monodisperse nonaggregated and nonagglomerated silica nanoparticles. Nanocluster(NC)- loosely bound agglomerates of nanosized paricles. The primary particle size of this NC filler ranges from 2 to $20 \mathrm{~nm}$, while the spheroidal agglomerated particles have a broad size dis $\rightarrow$ tribution, with an average particle size of 0.6 micro meters. Combination of two types of nanofillers result in the best combination of physical

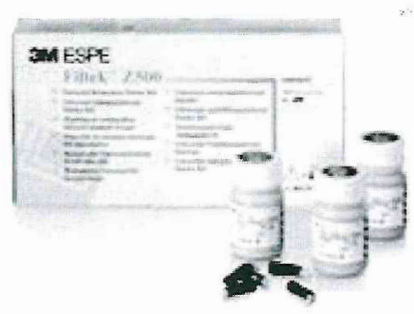

Figure 3 : Nanocomposite properties with superior esthetics, hence it can be used both as anterior and posterior restorative material.5 Example Filtek Supreme (3M ESPE)

Impression materi-

als- Nanofillers are integrated in vinylpolysiloxanes, producing a unique addition of siloxane impression materials. The material has better flow, improved hydrophilic properties and enhanced detail precision. Example Elite H-D from company Zhermack ${ }^{6}$

Periodontics - $\mathrm{TiO} 2$ nanotechnolgy can be used for melanin removal, incision of soft tissue without anaesthesia and periodontal therapy. Further nano-hydroxyapatite bone graft materials have now been developed to achieve regeneration of the lost periodontal bone around a natural tooth. ${ }^{7}$ This bone graft is superior to the other conventional materials in that it provides better binding of the newly formed bone with the underlying host bone.(Fig. 4) Also recently the dental implants have been developed with a nano titanium surface which is proposed to bind well to the host bone and provide a better osseointegration and thus improving the success of this treatment modality to replace missing teeth with the most naturalistic approach.

Bonding agents- $\mathrm{G}$ bond (Fig. 5) is a one step adhesive system. The interface formed by $\mathrm{G}$-bond is totally different from that of the interface formed by earlier bonding materials. The surface of the dentin is decalcified slightly, and there is almost no exposure of collagen fibers. This suggests that an extremely thin (300nm or less) interface is formed and that in this area, functional monomers contained in the bonding material react with hydroxyapatite at the 'nano' level to form insoluble calcium. This interface is expected to be stronger and more durable than that formed by other bonding materials. Prime \& Bond and Adper single bond plus adhesive also contains nanofillers which enters into dentinal

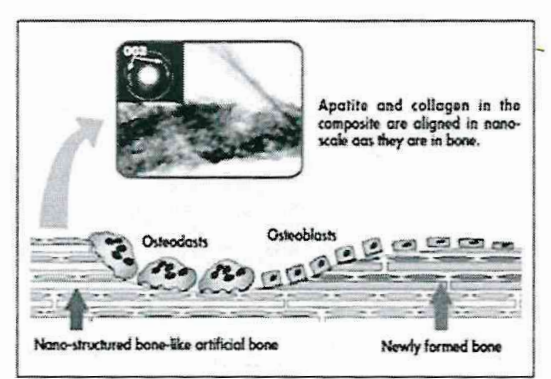

Figure 4 : Better bone binding observed with Nano bone graft materials

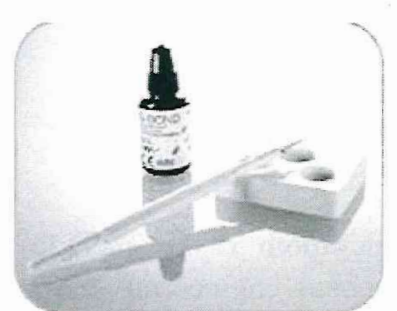

Figure 5 : Bonding agent ontaining Nano filler particles tubules to provide nano-retention.

Nano Glass Ionomer restorative- Generally glass ionomer restoratives can contain a broad range of particle sizes. Filler particle size can influence 


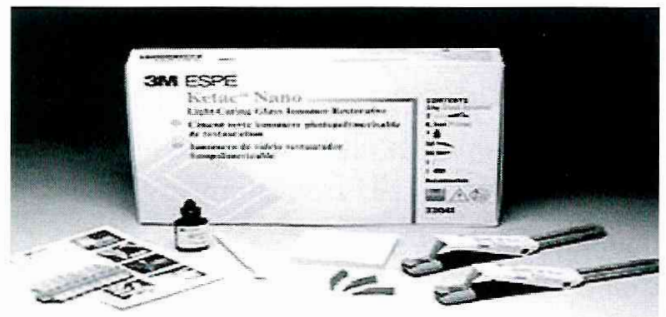

Figure 6: Nano glass ionomer cement

strength, optical properties, and abrasion resistance. By using bonded nanofillers and nanocluster fillers, along with fluoroalumino silicate glass newer type of GIC has been formulated using nanotechnology, provides better wear resistance and highly polished surface. Example Ketac Nano8 (3M ESPE) (Fig. 6)

Bone replacement materials - Hydroxyapatite nanoparticles used to treat bone defects are Ostim ${ }^{\circledR}$ - HA; VITOSSO - HA + TCP; NanOSSTM - HA. These can be used in maxillofacial injuries requiring bone graft, cleft patient and osseous defect in periodontal surgeries. ${ }^{9}$

Future treatment opportunities in Nanodentistry may include: Applications of nanorobots.

When the first micrometersized dental nanorobots are constructed, perhaps 10 to 20 years from today, how might they be applied to dentistry? Medical nanorobots might use specific motility mechanisms to crawl or swim through human tissues with navigational precision; acquire energy, and sense and manipulate their surroundings; achieve safe cytopenetration and use any of a multitude of techniques to monitor, interrupt or alter nerve-impulse traffic in individual nerve cells. These nanorobotic functions may be controlled by an onboard nanocomputer that executes preprogrammed instructions in response to local sensor stimuli. Alternatively, the dentist may issue strategic instructions by transmitting orders directly to in vivo nanorobots via acoustic signals (as are used in ultrasonography) or other means similar to an admiral commanding a fleet. ${ }^{1}$ (Fig. 7)

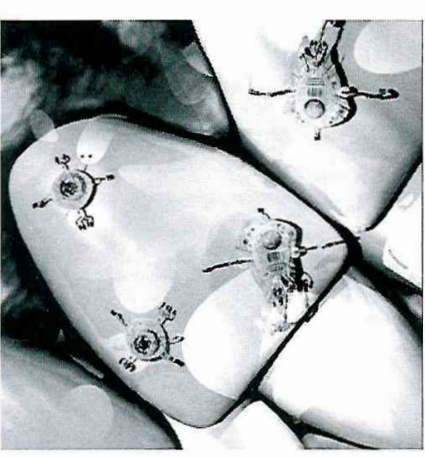

Figure 7 : Nanorobot

Tooth repair- Nanorobotic tooth repair includes several steps, first using genetic engineering, tissue engineering and tissue regeneration, and later involving the growth of whole new teeth in vitro and their installation. Ultimately there is installation of a biologically autologous whole replacement tooth that includes both mineral and cellular components, that is, complete replacement therapy should become feasible with time. ${ }^{1}$

Tooth renaturalization- Provides perfect treatment methods for esthetic dentistry. In this all fillings, crowns and other modifications to the visible dentition are removed with the affected teeth manufactured to become indistinguishable from original teeth. ${ }^{10}$

Hypersensitivity cure- Reconstructive dental nanorobots, using native biological materials, could selectively and precisely occlude specific tubules within minutes, offering patients a quick and permanent cure. ${ }^{1}$

Tooth repositioning- Orthodontic nanorobots could directly manipulate the periodontal ligament, cementum and alveolar bone allowing rapid and painless tooth repositioning within minutes to hours. ${ }^{11}$

Dental durability and cosmetics - Durability and appearance of tooth may be improved by replacing upper enamel layers with covalently bonded artificial material such as sapphire or diamond. Pue sapphire and diamond are brittle and prone to fracture, can be made more fracture resistant as part of a nanostructured composite material that possibly includes embedded carbon nanotubules.

Nanorobotic dentrifice - Nanorobotic dentifrice (dentifrobots) delivered by mouthwash or toothpaste could patrol all supragingival and subgingival surfaces at least once a day metabolizing trapped organic matter into harmless and odorless vapors and performing continuous calculus debridement. Properly configured dentifrobots could identify and destroy pathogenic bacteria residing in the plaque and elsewhere. ${ }^{12}$

Anesthesia - to induce oral anesthesia in the era of nanodentistry, dental professionals will instill a colloidal suspension containing millions of active analgesic micrometer-sized dental nanorobot particles on the patients gingivae and these ambulating nanorobots reaches dentin and by migrating into the gingival sulcus and passing painlessly through the lamina propria or the 1 - to $3-\mu \mathrm{m}$-thick layer of loose tissue at the cementodentinal junction.13 On reaching the dentin, the nanorobots enter dentinal tubule holes that are 1 to $4 \mu \mathrm{m}$ in diameter and proceed toward the pulp, guided by a combination of chemical gradients, temperature differentials and even positional navigation, all under the control of the onboard nanocomputer, as directed by the 
dentist. Once installed in the pulp and having established control over nerve-impulse traffic, the analgesic dental nanorobots may be commanded by the dentist to shut down all sensitivity in any tooth that requires treatment. ${ }^{1}$

Diagnosis and treatment of oral cancer - Nano electromechanical system (NEMS) converts biochemical to electrical signal and cantilever array sensor is an ultrasensitive mass detection technology that can be used for the detection of 10-12 bacteria, viruses and DNA. Drug delivery system that can cross the blood brain barrier is vision of the future with this technology. Parkinson disease, Alzheimer disease, brain tumors will be managed more efficiently. Nanovectors for gene therapy are in a developing stage to correct disease at molecular aspect. 9

Other applications could be local drug delivery, molecular monitoring, cutting of enamel and dentin etc.

\section{POTENTIAL RISKS OF NANOTECHNOLO- GY}

Nanopollution is a generic name for all waste generated by nanodevices or during the nanomaterials manufacturing process. This kind of waste may be very dangerous because of its size. It can float in the air and might easily penetrate animal and plant cells causing unknown effects. Most human-made nanoparticles do not appear in nature, so living organisms may not have appropriate means to deal with nanowaste. It is probably one great challenge to nanotechnology: how to deal with its nanopollutants and nanowaste. In free form nanoparticles can be released in the air or water during production (or production accidents) or as waste by-product of production, and ultimately accumulate in the soil, water or plant life. In fixed form, where they are part of a manufactured substance or product, they will ultimately have to be recycled or disposed of as waste. It is not known yet whether certain nanoparticles will constitute a completely new class of non-biodegradable pollutant. ${ }^{14}$

\section{CONCLUSION}

The vision described above may sound unlikely, implausible, or even heroic. Yet, the theoretical and applied research to turn them into reality is progressing rapidly. This science may now seem to be a fiction. But, looking at the progress made in this field since Feynman saw the first vision of nanoscience in 1959 we can definitely expect a 'NANO TSUNAMI' to strike us in near future. Nanodentistry will give a new visionary to comprehensive oral health care, as now trends of oral health have been changing to more preventive intervention than a curative $\&$ restorative procedure. Nanodentistry has strong potential to transfigure dentists to diagnose and treat disease in future. Though in its infancy, nanotechnology is bound to change dentistry, health care and human life more profoundly than other developments. In order to prevent any potential adverse environmental impacts, proper evaluation, including full-scale ecosystem-wide studies, of these nanoparticles needs to be addressed before this technique is used on a mass scale.

\section{REFERENCES}

1. Freitas RA Jr. Nanodentistry. J Am Dent Assoc 2000; 131: 1559-1566.

2. Schleyer TL. Nanodentistry - Fact or Fiction? J Am Dent Assoc 2000; 131: 1567-1568.

3. Patil M, Mehta DS, Guvva S. Future impact of nanotechnology on medicine and dentistry. $J$ Indian Soc Periodontol 2008;12:34-40.

4. Saunders SA. Current practicality of nanotechnology in dentistry. Part I: Focus on nanocomposite restorative and biomimetics. Clinical, Cosmetic and Investigational Dentistry 2009:1 47-61.

5. Mitra S, Wu D, Holmes B.N. An application of nanotechnology in advanced dental materials. J Am Dent Assoc 2003 Oct; 134: 1382-90.

6. Rybachuk AV , Chekman IS, Nebesna TY. Nanotechnology and nanoparticles in dentistry. Pharmacology And Pharmaceutics 2009; 1: 18-21.

7. Tran N, Webster TJ. Nanotechnology for bone materials. Wiley Interdiscip Rev Nanomed Nanobiotechnol. 2009 May; 1(3):336-51

8. Ketac ${ }^{\mathrm{TM}}$ Nano Light-Curing Glass Ionomer Restorative. $\quad \mathrm{http} / / /$ solutions.3m.com/wps/portal/3M/en_US/3M-ESPE/dental- professionals/products/category/direct-restorative/ketac-nano/

9. Verma SK, Prabhal KC, Goyal L, Rani M, Jain A. A critical review of the implication of nanotechnology in modern dental practice. Natl J Maxillofac Surg 2008;1:41-44.

10. ADA Council on Scientific Affairs. Dental amalgam: update on safety concerns. J Am Dent Assoc 1998; 129: 494-503.

11. Shellhart WC, Oesterle LJ. Uprighting molars without extrusion. J Am Dent Assoc 1999;130:381-385.

12. Freitas RA Jr. Nanomedicine. Basic capabilities, Georgetown, TX; Landes Bioscience 1999;1:345-350.

13. Paulsen F, Thale A. Epithelial-connective tissue boundary in the oral part of the human soft palate. J Anat 1998; 193:457-467.

14. Environmental implications of nanotechnology. www.wikipedia.org/wki/nanotechnology. 